\title{
Investigation on the Tensile Behavior of Graphene-Aluminum Nano-Laminated Composites by Molecular Dynamics Simulation
}

\author{
Jia-Qi Zhu, Qing-Sheng Yang ${ }^{*}$, Xia Liu \\ Department of Engineering Mechanics, Beijing University of Technology, Beijing, 100124, China \\ *Corresponding author, Email: qsyang@bjut.edu.cn
}

Keywords: Graphene/aluminum composite; Tensile behavior; Dislocation; Yield stress; Yield strain

\begin{abstract}
Graphene-aluminum (Gr/Al) composite laminated by aluminum (Al) and graphene sheets alternately has excellent mechanical properties thanks to the high strength, high Young's modulus and the two-dimensional atomic structure of graphene. In this study, the uniaxial tensile properties of Gr/Al nano-laminated composite are studied by molecular dynamics (MD) method. It is found that the thickness of Al layer has a significant effect on the tensile strength and Yang's modulus of the $\mathrm{Gr} / \mathrm{Al}$ composite. Composite with a smaller thickness of Al layer shows better properties. Graphene not only block propagation of dislocations, but bear most of the loads, resulting in higher Young's modulus, tensile strength and failure strain of the composites than those of pure Al. The simulation of temperature-effect shows that the $\mathrm{Gr} / \mathrm{Al}$ composite is difficult to arise plastic deformation at low temperature, which lead to a higher strength and modulus of the composite. In addition, the effect of graphene stacking on the properties of composites is investigated. Through tensile tests at the vertical and parallel interfaces, it is found that graphene stacking may lead to a reduced performance of the composite.
\end{abstract}

\section{Introduction}

Controlling the microstructure of the materials appropriately can make a remarkable enhancement in the mechanical properties of metals. This is an effective method to promote the development of structural materials. Metal matrix composites (MMCs) reinforced by graphene and its derivatives with laminated microstructure have attracted much attention due to their excellent properties such as high strength, Young's modules and good ductility [1,2]. As a single-atomic-layer material, graphene may form strong interface bonding with metal and enhance the properties of the composite due to the large surface area and extraordinary mechanical properties [3].

Recently, graphene reinforced metals nano-laminated composite has been studied by many researchers since it was successfully prepared [4]. Al reinforced by few layers of graphene was fabricated by Shin et al. and the strengthening behavior of the Gr/Al composite was investigated through tensile test. It was found that the tensile strength of the composite reached two times that of pure $\mathrm{Al}$ (up to $440 \mathrm{MPa}$ ) with only $0.7 \mathrm{vol} \%$ graphene addition [5]. Li et al. [6] studied the tensile behavior of the Gr/Al bioinspired laminated composite. They found that graphene in the nanolaminated structure can effectively improve the strength and hardness of composites without reducing the plasticity. Subsequently, the compression experiments on micro-pillars done by Feng et al. [7] showed that the arrangement of graphene was a critical factor affecting the strength of composite, so the importance of structural design is emphasized. Besides the experiments, MD simulation was also used in previous studies to investigate the enhancement effect of graphene on metal. The single-layer and double-layer graphene reinforced copper showed a higher tensile strength and failure strain than pure metal, as reported by Duan et al. [8] Recently, a graphene/copper composite with obvious anisotropic property was fabricated experimentally. It is suggested that both the tensile strength and elongation at in-plane direction are better than that of through-plane direction. [9]. The elastic-plastic deformation mechanism of metal/graphene nano-layered composites under tensile loading is investigated and they found graphene can provide an effective barrier to dislocation propagation. Although there has been many studies on the enhancement effect of graphene on metals, the investigation on the graphene aggregation which will reduce the mechanical behavior is still lack, it 
is not enough to fully understand the strengthening mechanism of the presence of graphene and the weakening mechanism of the stacked graphene structure.

In this study, the mechanical properties of $\mathrm{Gr} / \mathrm{Al}$ nano-laminated composite under tensile loading are investigated using the MD simulation. It was found that the stacked graphene structure will extremely weaken the performance of the composite. The different spacing distance of graphene and the different temperature is also considered based on the stress-strain curves and the total length of dislocation lines. In addition, the atomic structure and the local strain distribution during the tension process are plotted to give a better explanation of the deformation and reinforcement mechanism.

\section{Models and Method}

The schematic of the Gr/Al nano-laminated composite is shown in Fig. 1(a). The studied representative volume element (RVE) of Gr/Al composite used as the MD model taken from the bulk composite is shown in Fig. 1(b). The coordinate systems of the arrangement of Al lattice are set to $x$ [100], $y$ [010] and $z$ [001]. The initial dimension of the RVE is a cube with a length of $10 \mathrm{~nm}$. The thickness of the stacked graphene and the interlayer spacing of the graphene layers are represented $t$ and $d$, respectively. The value of $t$ is determined by the number of stacking layers of graphene and its initial value is $0.34 \mathrm{~nm}$ with monolayer of graphene. In order to eliminate the boundary effect caused by the limitation of simulation system, periodic boundary conditions are applied in all the three directions.

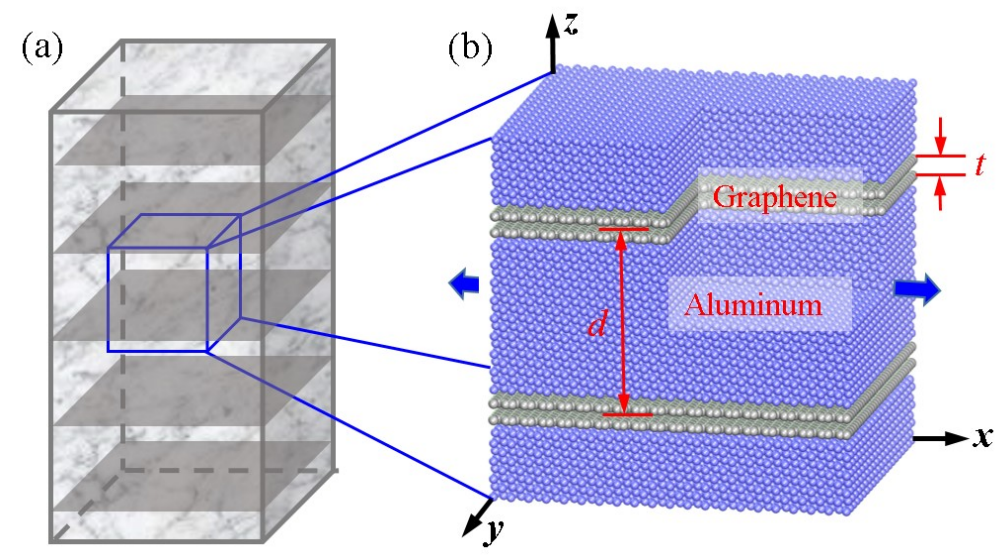

Fig. 1 (a) Schematic of the nanolaminated Gr/Al nano-pillar. (b) The RVE taken from the nanopillar for compression property investigation - marked with the blue box

All the simulations on Gr/Al nanocomposite are performed using LAMMPS (Large-scale Atomic/Molecular Massively Parallel Simulator). Interactions between $\mathrm{Al}$ and $\mathrm{Al}$ atoms were modelled using the embedded atom model (EAM) potential. The adaptive intermolecular reactive empirical bond order (AIREBO) potential [10]was used to model the interactions between graphene atoms. Besides, the van der Waals interactions were modelled using the 12-6 Lennard-Jones (LJ) potential and the parameters for the $\mathrm{C}$-Al interface are $\varepsilon=0.035078 \mathrm{eV}$ and $\sigma=3.0135 \AA \AA$ [11]. First, the whole system was subjected to an energy-minimization process with a specified energy and the force tolerance of $1 \times 10-12$ by iteratively adjusting the atom coordinates. Then the configuration will be in a stable stage with a low potential energy. Subsequently, the system relaxed under the NPT ensemble. Constant temperature and pressure are controlled by the Nose-Hoover thermostat and barostat. After the equilibration, a uniaxial tension of the composite was carried out by expanding the simulation box at a strain rate of $0.01 \mathrm{ps}^{-1}$ under a NVE ensemble. The timestep for one integration was set to $0.001 \mathrm{ps}$. The temperature was maintained at a room temperature by explicitly rescaling the velocities of the atoms during the simulation. After the simulations, we used the dislocation extraction algorithm (DXA) function of the Open Visualization Tool (OVITO) [12] to analyze the dislocations within the deformed structures. 


\section{Results and Discussions}

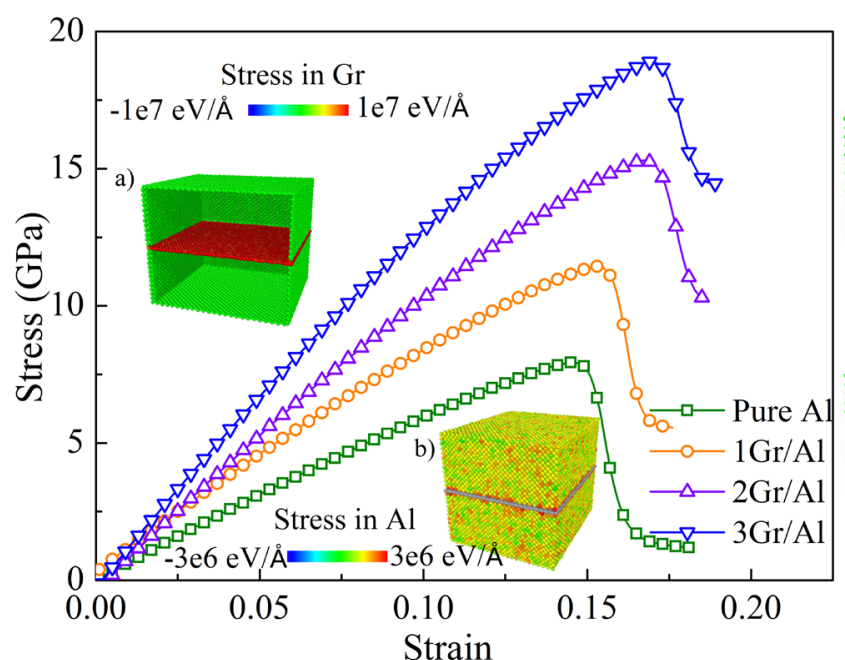

(a)
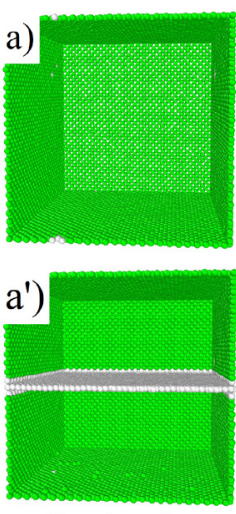

Strain $=0$
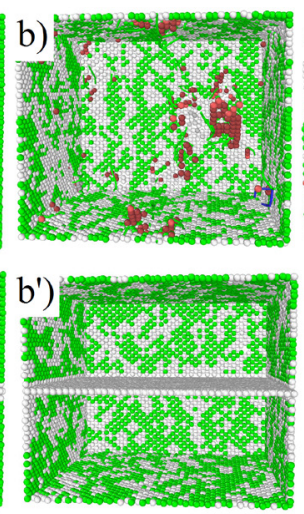

Strain $=0.15$
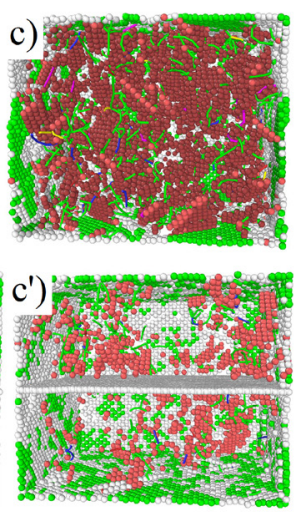

Strain $=0.16$

(b)

Fig. 2 Tension simulation of the pure $\mathrm{Al}$ and $\mathrm{Gr} / \mathrm{Al}$ nanocomposite. (a) Stress-strain curves of the composite with different interlayer spacing distance, (b) Atomic structure distribution (the green atom represents the fcc structure, and the red atom represents the hcp structure).

In this work, Al reinforced by $n$ layers of single-layer garphene are represented by $n \mathrm{Gr} / \mathrm{Al}$ while the Al reinforced by $n$ layers of stacked graphene are represented by $n \# \mathrm{Gr} / \mathrm{Al}$. Different number of dispersed graphene layers means different interlayer spacing distance ( $n$ equals 1, 2 and 3 indicating interlayer spacing of $10 \mathrm{~nm}, 5 \mathrm{~nm}$ and $3.3 \mathrm{~nm}$, respectively). The stress-strain curves of the pure Al and $\mathrm{Gr} / \mathrm{Al}$ nano-laminated composite under tension loading are plotted in Fig.2(a) and the distribution of the tensile stress is inserted in it. All the curves increase linearly at the initial stage, corresponding to the elastic deformation stage of the material when the stress-strain relationship obeys Hooke's law. Then the curves rise slowly to the maximum stress point, corresponding to the plastic stage of the material due to the generation of dislocations. After reaching the peak point, the stress drops rapidly, which means the yield of the composite. It can be clearly seen that the Young's modules, tensile strength and the yield strain increased significantly compared with pure Al. The inserted picture in Fig. 2(a) shows the stress distribution of $x$ direction at the strain of 0.15 . It can be seen that graphene bears much greater load than Al. This is due to the inherent high strength and modulus of graphene which contributes greatly to the strengthening and toughening of the $\mathrm{Gr} / \mathrm{Al}$ nano-laminated composite.

Fig. 2(b) shows the atomic structure during the tension loading. All atoms retain their original fcc structure before loading-see Fig. 2(b) a)-a'). In the pure Al model, the disloctions occurred at a low strain of 0.15 and expand across the whole model without truncation - corresponding to picture $b$ ) and c) in Fig.2(b). While in the Gr/Al nanocomposite, dislocations occurred at a higher strain of 0.16, which means that the Al reinforced by graphene has a longer elastic deformation stage without generation of any dislocation. When the strain reached 0.16 , dislocations generated gradually at the internal of the $\mathrm{Al}$ and the interface between $\mathrm{Gr}$ and $\mathrm{Al}$. It is clearly that the dislocation is blocked and stacked at the interface, which is critical to the strengthening of the composite - corresponding to picture b') and c') in Fig.2(b).

In order to explore the disadvantage of the graphene aggregation in G/Al nanocomposite, the stress-strain curves of stacked graphene and dispersed gaphene reinforced $\mathrm{Al}$ are plotted together, as shown in Fig. 3(a). Although the presence of graphene can enhance the mechanical performance of $\mathrm{Al}$, the enhancement effect of stacked graphene reinforced Al is worse than that of dispersed graphene. For example, with the same graphene content, the tensile strength and yield strain of the $2 \mathrm{Gr} / \mathrm{Al}$ composite is $16.5 \mathrm{GPa}$ and 0.17 , respectively. However, the tensile strength and yield strain of the $2 \# \mathrm{Gr} / \mathrm{Al}$ composite is $14.8 \mathrm{GPa}$ and 0.16 , respectively, which are all lower than that of $2 \mathrm{Gr} / \mathrm{Al}$ composite. 


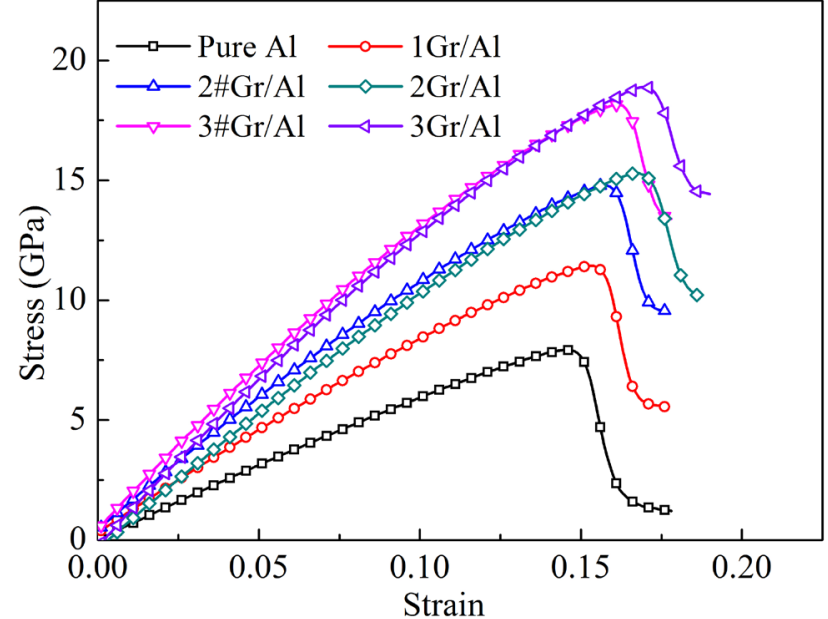

(a)

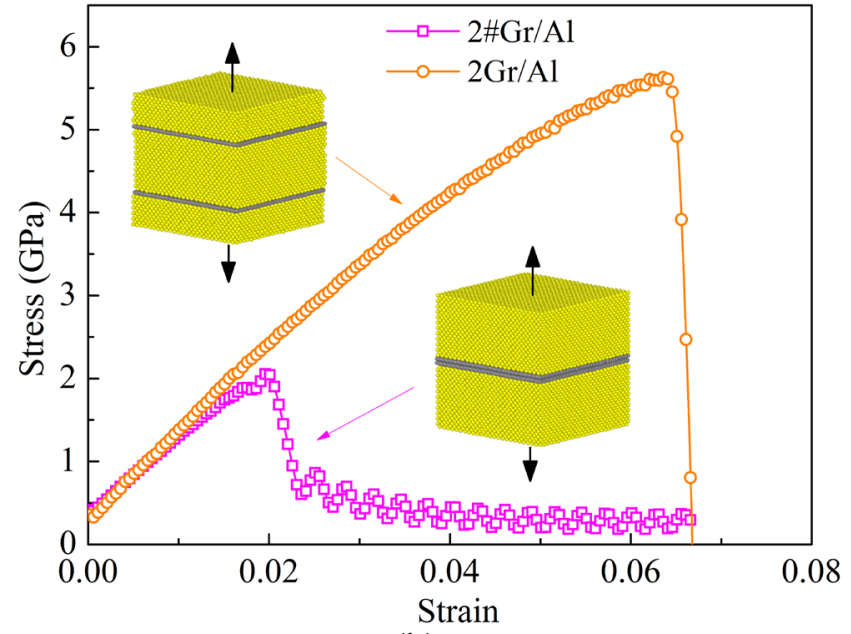

(b)

Fig. 3 Tension simulations on the $\mathrm{Al}$ reinforced by stacked graphene and dispersed graphene. (a) Stress-strain curves of Gr/Al nanocomposite under in-plane tension, (b) Stress-strain curves of $\mathrm{Gr} / \mathrm{Al}$ nanocomposite under out-plane tension.

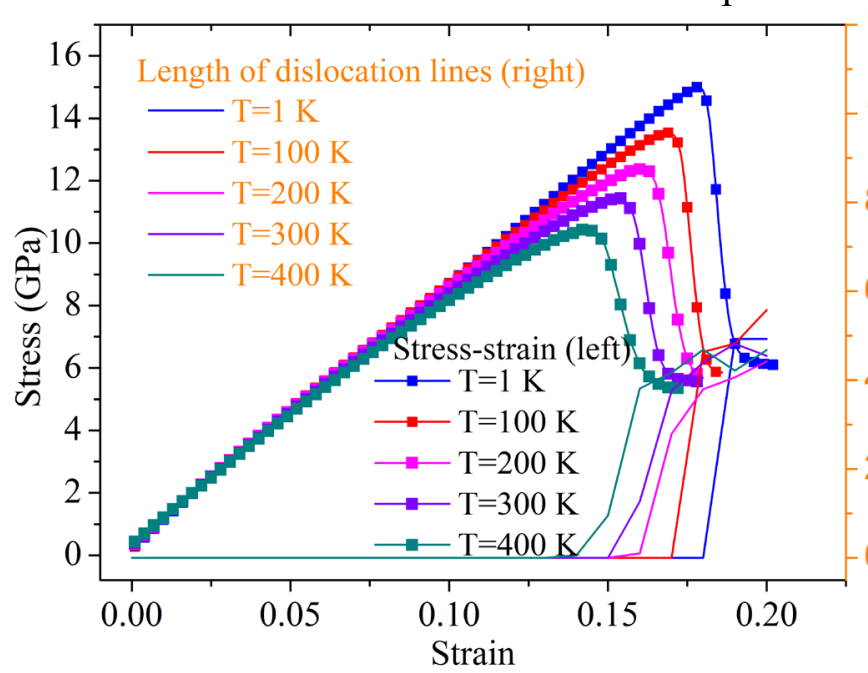

(a)

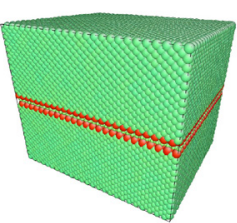

$1 \mathrm{~K}$

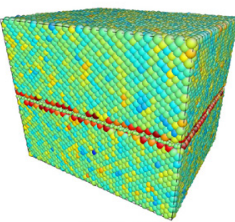

$300 \mathrm{~K}$

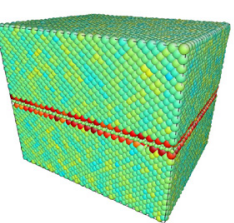

$100 \mathrm{~K}$

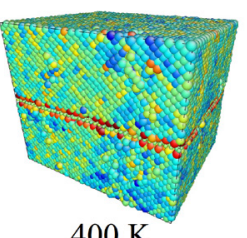

$400 \mathrm{~K}$

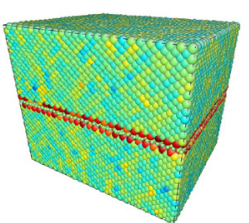

$200 \mathrm{~K}$

0.23

Strain

(b)

Fig. 4 Tension simulations on the Gr/Al composite under different temperature. (a) Stress-strain curves of $\mathrm{Gr} / \mathrm{Al}$ nanocomposite and the variation of the length of dislocation lines, (b) Distribution of the local plastic deformation at a strain of 0.16 under different temperature.

In additon, the tensile simulation of vertical interface are also performed to investigated the outplane properties of the Gr/Al nanocomposite and the result is shown in Fig.3(b). The main reason for the great difference between the $2 \mathrm{Gr} / \mathrm{Al}$ and $2 \# \mathrm{Gr} / \mathrm{Al}$ is the different failure part. During the tension process, the failure of the $2 \mathrm{Gr} / \mathrm{Al}$ is caused by the separation between $\mathrm{Al}$ and $\mathrm{C}$ atoms, while the failure of the $2 \# \mathrm{Gr} / \mathrm{Al}$ is due to the separation of $\mathrm{C}$ and $\mathrm{C}$ atoms. It is proved in previous study[3] that the interaction between $\mathrm{Al}$ and $\mathrm{C}$ atoms is stronger than that of $\mathrm{C}$ and $\mathrm{C}$ atoms. Therefore, it can be concluded that the stacked graphene structure will decrease the mechanical properties of the $\mathrm{Gr} / \mathrm{Al}$ composite as mentioned in the former research [13] and the results are in accordance with the experimental result.

Finally, the temperature-effect on the mechanical performance was investigated based on the total length of dislocation lines and the local strain distribution of the composite. Fig. 4(a) shows the stressstrain curves and the length of dislocation lines under different simulation temperature. It is found that temperature has no effect on the Young's modulus, but the tensile strength and yield strain decrease with the increase of temperature. The local strain distribution of the $1 \mathrm{Gr} / \mathrm{Al}$ composite at a strain of 0.16 is shown in Fig. 4(b). It is obvious that the local plastic deformation is more serious under a high temperature. This is because the thermal fluctuation wherein the kinetic energy of atoms increase at a high temperature and result in a worse performance of the composite. 


\section{Summary}

In this study, the mechanical properties of the Gr/Al nano-laminated composite under tension loading was investigated using molecular dynamics method. The simulation results were analyzed base on the stress-strain curves, length of the dislocation lines, the atomic structure and the distribution of local plastic deformation. It is found that the $\mathrm{Al}$ reinforced by few layers of graphene has a significant higher performance than pure $\mathrm{Al}$ due to the dislocation-blocking mechanism at the interface. Besides, the graphene can bear most of the stress which will contribute to the enhancement of the nano-laminated composite. Subsequently, the worse mechanical properties along out-plane direction cased by the stacked graphene structure is studied. The result shows that the reason for the worse performance is that the interaction between $\mathrm{C}$ and $\mathrm{C}$ atoms is weaker than that of $\mathrm{Al}$ and $\mathrm{C}$ atoms. In addition, the uniaxial tension of the $\mathrm{Gr} / \mathrm{Al}$ composite under different temperature is also investigated to discuss the temperature-effect. It is found that the $\mathrm{Gr} / \mathrm{Al}$ composite shows a better performance at a low temperature. The Young's modules, tension strength and the yield strain will decrease with the increase of the temperature because the thermal fluctuation.

\section{Acknowledgements}

This work is supported by the Natural Science Foundation of China $(11502007,11472020,11772012$, 11872079 and 11632005), and the General Program of Science and Technology Development Project from Beijing Municipal Education Commission (KM201810005002).

\section{References}

[1] Z. Hu, G. Tong, D. Lin, C. Chen, H. Guo, J. Xu, et al., Graphene-reinforced metal matrix nanocomposites - a review, Mater Sci Tech-Lond. 32 (2016) 930-53.

[2] M. Singh, R.S. Rana, R. Purohit, K. Sahu, Development and analysis of Al-matrix nano composites fabricated by ultrasonic assisted squeeze casting process, Mater Today: Proc. 2 (2015) 3697-703.

[3] K. Duan, L. Li, Y. Hu, X. Wang, Interface mechanical properties of graphene reinforced copper nanocomposites, Mater Res Express. 4 (2017) 115020.

[4] Y. Kim, J. Lee, M.S. Yeom, J.W. Shin, H. Kim, Y. Cui, et al., Strengthening effect of singleatomic-layer graphene in metal - graphene nanolayered composites, Nat Commun. 4 (2013) 2114.

[5] S.E. Shin, H.J. Choi, J.H. Shin, D.H. Bae, Strengthening behavior of few-layered graphene/aluminum composites, Carbon. 82 (2015) 143-51.

[6] Z. Li, Q. Guo, Z. Li, G. Fan, D. Xiong, Y. Su, et al., Enhanced mechanical properties of graphene (reduced graphene oxide)/aluminum composites with a bioinspired nanolaminated structure, Nano Lett. 15 (2015) 8077-83.

[7] S. Feng, Q. Guo, Z. Li, G. Fan, Z. Li, D. Xiong, et al., Strengthening and toughening mechanisms in graphene-Al nanolaminated composite micro-pillars, Acta Mater. 125 (2017) 98-108.

[8] K. Duan, F. Zhu, K. Tang, L. He, Y. Chen, S. Liu, Effects of chirality and number of graphene layers on the mechanical properties of graphene-embedded copper nanocomposites, Comp Mater Sci. 117 (2016) 294-9.

[9] K. Chu, F. Wang, X. Wang, D. Huang, Anisotropic mechanical properties of graphene/copper composites with aligned graphene, Materials Science and Engineering: A. 713 (2018) 269-77.

[10] D.W. Brenner, O.A. Shenderova, J.A. Harrison, S.J. Stuart, B. Ni, S.B. Sinnott, A secondgeneration reactive empirical bond order (REBO) potential energy expression for hydrocarbons, $\mathrm{J}$ Phys-Condens Mat. 14 (2002) 783-802. 
[11] N. Silvestre, B. Faria, J.N. Canongia Lopes, Compressive behavior of CNT-reinforced aluminum composites using molecular dynamics, Compos Sci Technol. 90 (2014) 16-24.

[12] A. Stukowski, Visualization and analysis of atomistic simulation data with OVITO-the Open Visualization Tool, Model Simul Mater Sc. 18 (2010) 2154-62.

[13] K. Chu, C. Jia, Enhanced strength in bulk graphene-copper composites, physica status solidi (a). 211 (2014) 184-90. 